\title{
Long term Immunogenicity of Single Dose of Live Attenuated Hepatitis A Vaccine in Indian Children - Results of 15-Year Follow-up
}

\author{
Sheila Bhave ${ }^{1}$, Amita Sapru ${ }^{1}$, Ashish BavdekaR ${ }^{1}$, Rishi Jain ${ }^{2}$, Khokan Debnath, ${ }^{2}$ VaibHaVi Kapatkar ${ }^{2}$ \\ From ${ }^{1}$ Department of Pediatrics, KEM Hospital Research Centre, Pune and ${ }^{2}$ Department of Medical Affairs, Wockhardt Limited, \\ Mumbai; Maharashtra.
}

Correspondence to: Dr Sheila Bhave, Consultant in Pediatric Research, Department of Pediatrics, KEM

Hospital Research Centre, Rasta Peth, Pune 411 011, India.

peds@kemhrcpune.org

Received: July 07, 2020;

Initial review: August 28, 2020;

Accepted: February 12, 2021
Objectives: To measure anti-HAV antibodies 15 years after a single dose of live attenuated hepatitis A vaccine in Indian children. Methods: Of the 143 children vaccinated in 2004, 109 were evaluated in 2019, clinically and for anti-HAV antibodies. These children have been assessed clinically every year, and for anti-HAV antibodies in 2004, 2007, 2010 and 2014. Results: Of the 109 children who came for the present assessment, 11 had received additional doses of hepatitis A vaccine in 2004/2007 because of low anti-HAV titre (<20 mlU/ $\mathrm{mL})$. In the remaining 98 children, $94(96 \%)$ had seroprotective levels with a geometric mean titre of $79.6 \mathrm{mIU} / \mathrm{mL}$. Seroprotection rate in all 109 children was $86.2 \%$. Conclusions: Single dose of live attenuated hepatitis A vaccine in Indian children demonstrated robust immunogenicity at 15 years post vaccination.

Keywords: Hepatitis A vaccine, Anti-HAV anti bodies, Immune memory, Safety.

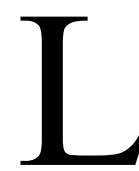
ive attenuated hepatitis A vaccine (H2 strain) has a long history of development and research (in China) for nearly three decades $[1,2]$. Promising immunogenicity, safety and protection have been reported, using a single dose of the vaccine [3]. In India, a live vaccine was licensed in 2005 and has been used extensively since then. Immuno-genicity studies of the single dose regimen in India have matched the Chinese reports $[3,4]$. Both, World Health Organization (WHO) and Indian Academy of Pediatrics (IAP) have endorsed the single dose schedule of live hepatitis A vaccine in the routine immunization of children aged one year or above $[5,6]$.

In this study, we report the anti-HAV antibodies at 15 years from the first Indian study of single dose live HAV vaccine in children. Immunogenicity data from the same cohort at 2 months, 30 months and 10 years postimmunization has previously been reported [7-9].

\section{METHODS}

The study began in 2004 wherein 143 children were given a single dose of $\mathrm{H} 2$ strain of a live attenuated hepatitis $\mathrm{A}$ vaccine (Biovac-A, Wockhardt Ltd) and assessed for anti-HAV antibodies 2-months post vaccination [7]. These children were then called for follow-up every year, for clinical assessment and to record history of hepatitis, if any. They were assessed serially for anti-HAV antibodies in 2007, 2010 and 2014 [8,9]. Subjects with low anti-HAV antibody titres $(<20 \mathrm{mIU} / \mathrm{mL})$ were given additional doses of vaccine viz., in 2004 they were given two doses of the then licensed HAV vaccine (Havrix $\mathrm{Jr}$ GSK Biologicals) and in 2007 they received an additional dose of Biovac-A vaccine. No further vaccines were given in 2010 or 2014.

Contact details of the cohort were maintained by medical social workers. At the yearly visits, participants/ parents were asked for history of hepatitis like illness (fever, anorexia, nausea, vomiting and jaundice). Clinical examination included noting for hepatomegaly or splenomegaly, if any. Parents were reminded to report complaints of hepatitis like illness immediately. No diary was given to participants for recording signs and symptoms. In the present study (2019) too, these children were clinically assessed for evidence of hepatitis (if any) and their anti-HAV antibodies were measured.

After routine clinical assessments, blood samples were collected and sent for total and IgM anti-HAV antibody analysis (Cobas anti-HAV electro-chemiluminescence immunoassay, ECLIA, Roche Diagnostics Deutschland $\mathrm{GmbH}$ ) to an independent accredited laboratory (SRL Diagnostics). Seroprotection rate was defined as proportion of subjects with total anti-HAV antibody level $\geq 20 \mathrm{mIU} / \mathrm{mL}$. Geometric mean titre (GMT) for anti-HAV antibodies was calculated as per standard method. Data was entered in predesigned paper case report forms (CRFs). All study documents were 


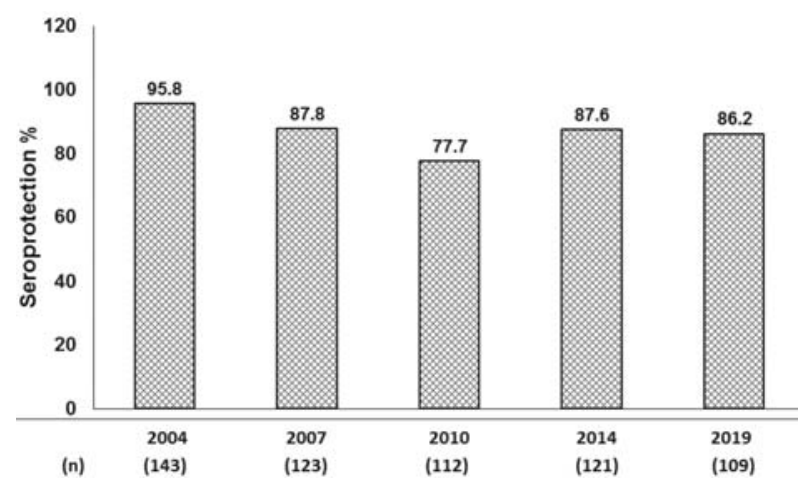

Fig. 1 Serial seroprotection rates over 15 years (\%).

maintained in a dedicated study cupboard with restricted access. Data analysis was done using Stata 13.1 (StataCorp). All analysis was done using two-sided tests at alpha 0.05 (95\% confidence level).

During the study of 15 years, institutional ethics committee approval and informed consent were obtained three times: Initial study (2004), second phase of study (2007-2015), and third phase of study (2016-2019). During the study, whenever a participant attained the age of 18 years, informed consent was obtained from him/her for continuing in the study.

\section{RESULTS}

Of the original 143 children who received a single dose of live attenuated hepatitis A vaccine in 2004, 109 subjects (72 males) came for the fifteen-year follow-up assessment in 2019. Mean age was 19.7 years (range 16-26.8 years). The number of subjects who came for follow-up since vaccination is as follows: 2.5 years $(n=131), 6$ years $(n=126), 10$ years $(n=121)$ and 15 years $(n=109)$. None

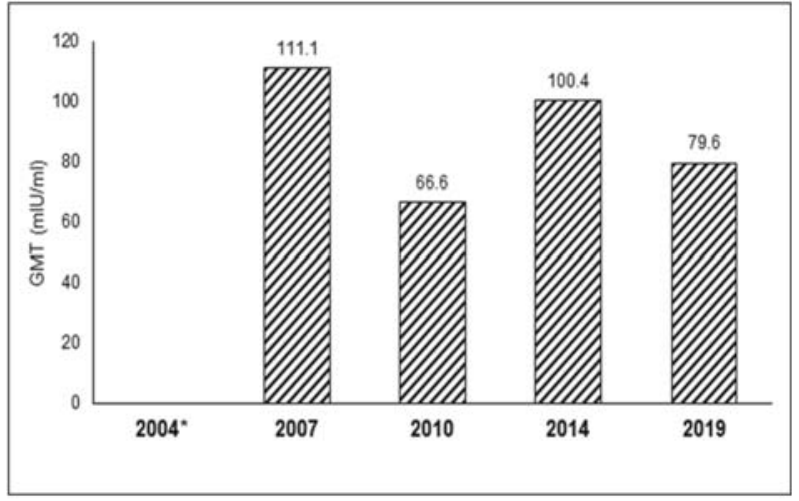

*Anti - HAV tires > 100 MIU/mL were not quantified in 2004, Hence GMT of 2004 not represented in figure. GMT-geometric mean titers.

Fig. 2 Serum anti-HAV antibody GMTs over 15 years. of the parents/children retracted consent in writing. Clinical examination of the participants did not reveal any abnormal findings, and none gave any history of hepatitis like illness in the past.

Of the 109 children who came for the present followup, 4 had received two doses of licensed inactivated HAV vaccine in 2004, and 7 others had received a second dose of live HAV vaccine in 2007, as their total anti-HAV antibody levels had dropped to $<20 \mathrm{mIU} / \mathrm{mL}$.

Of the remaining 98 children, 4 had low anti-HAV titres $(<20 \mathrm{mIU} / \mathrm{mL})$ giving a seroprotection rate of $95.9 \%$. If the 11 children who were given additional doses of HAV vaccine are also included, the seroprotection rate in all 109 children was $86.2 \%$. The comparison of sero-protection rate in 2019 with previous assessment years is shown in Fig. 1.

All children were found to be negative for anti-HAV IgM. The total anti-HAV geometric mean titre (GMT) in 'seroprotected children' who received single dose of live attenuated vaccine $(n=94)$ is $79.6 \mathrm{mIU} / \mathrm{mL}(95 \%$ CI 69.2-91.56). The comparison of GMT value at 15 years with previous assessment years is shown in Fig. 2.

In 2010, there were 25 children with anti-HAV titres $<20 \mathrm{mIU} / \mathrm{mL}$. They were not given any additional dose / doses of live/inactivated HAV vaccine. The serial antiHAV GMTs of these 25 children as compared to all 98 with single dose of live HAV vaccine is shown in Fig. 3. In 2014 and 2019, 23 of these 25 regained seroprotective levels. In 2019, the anti-HAV antibody titre of two other children (who had seroprotective levels earlier) are now $<20 \mathrm{mIU} / \mathrm{mL}$.

Children who received two additional doses of an inactivated HAV vaccine in $2004(n=4)$, or an additional

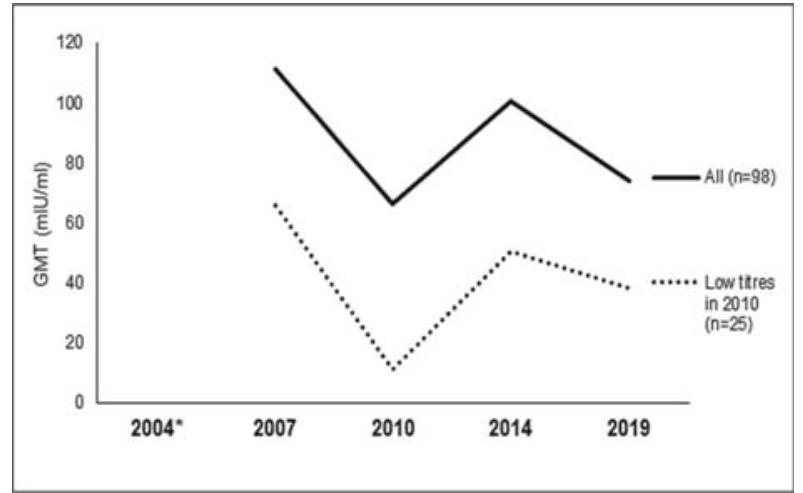

*Anti - HAV tires > 100 MIU/mL were not quantified in 2004, Hence GMT of 2004 not represented in figure. GMT-geometric mean titers.

Fig. 3 Serum anti-HAV antibody GMTs of children with low titers in 2010. 


\section{WHAT THIS STUDY ADDS?}

- A single dose of live attenuated hepatitis A vaccine shows robust immunogenicity with seroprotection level of $86.2 \%$ at 15 years after vaccination in Indian children

dose of live HAV vaccine in $2007(n=7)$ have continued to show seroprotective levels since additional vaccination.

\section{DISCUSSION}

This 15-year follow-up of a cohort of children vaccinated with a single dose of live attenuated HAV vaccine shows a seroprotective rate of $86.2 \%$ with anti HAV GMT value of $79.6 \mathrm{mIU} / \mathrm{mL}$. The serial seroprotection rates of the cohort are $95.8 \%$ at 2 months, $87.8 \%$ at 30 months, $77.7 \%$ at 6 years, $87.6 \%$ at 10 years and $86.2 \%$ at 15 years. This evaluation at 15 years confirms the robust long-term immunogenicity of a single dose of live HAV vaccine and compares well with other Indian and Chinese studies [3,10-12]. The comparable long term Chinese seroprotection data (Zhuang, et al.) at 15 years is $81.3 \%$ (GMT $128 \mathrm{mIU} / \mathrm{mL}$ ) [2,3]. The other Indian long term multicentric study reported an immunogenicity of $97.3 \%$ at 5 years with GMT of $127.1 \mathrm{mIU} / \mathrm{mL}$ [11].

In our serial evaluations since 2004, we used two types of immunoassay test kits: Axsym HAVB ELISA (Abbott Labs), (2004, 2007 and 2010). These kits were not available in India in 2014. Hence, we used COBAS kits based on ECLIA technology (Roche Diagnostics) in 2014 and 2019.The higher antibody titres of 2014 and 2019 could be due to differences in kits as ECLIA based reports are known to be of a higher sensitivity as compared to ELISA [13]. Alternatively, higher titres could be due to 'booster like' response to exposure to naturally occurring antigens of HAV in the community [14]. As the child grows from a teenager to adulthood, the frequency of consuming food and water outside the home increases, thereby increasing exposure to hepatitis A.

Another limitation of our study was cohort contamination with additional doses of hepatitis A vaccine [9]. In 2004, 6 children were given two doses of inactivated $\mathrm{HAV}$ vaccine and in the next evaluation at 30 months, 9 others were given a second dose of the live HAV vaccine. At the 6 yrs follow-up in 2010, 25 children were found to be seronegative. These 25 children were not given any additional doses of vaccine. Interestingly, 23 of these 25 were in the seroprotected range in the present evaluation, further implying the probability of an anamnestic response to natural boosters. Chen, et al. [15] have recently demonstrated that anamnestic responses via memory $\mathrm{B}$ and memory $\mathrm{T}$ cells may provide long term protection after a single dose of live Hepatitis A vaccine, despite low levels of anti-HAV antibodies.

In conclusion, 15-year follow-up after a single dose of live hepatitis A vaccine (H2 strain) demonstrated robust immunogenicity in Indian children. The continued safety and immunogenicity profile of the vaccine reiterates its value in primary immunization of Indian children. As a policy decision, the single dose schedule cuts costs while providing definitive long-term protection.

Acknowledgements: Dr Sonali Shah for data management and follow-up; Dr Deepak Langade (Clinsearch Healthcare Solutions Pvt Ltd) for statistical inputs; Dr Archana Karadkhele (Wockhardt Ltd) \& Dr Pramit Sonone (Wockhardt Ltd) for valuable inputs.

Ethics clearance: KEM Hospital Research Centre Ethics Committee; No. KEMHRC/LFG/ EC/778 dated June 6, 2016.

Contributors: SB, AS, AB: designed the study, recruited patients, analyzed results and wrote the manuscript; RJ, KD, VK: provided technical help needed for the study.

Funding: Wockhardt Ltd. Recipient of funds is KEM Hospital Research Centre, Pune.

Competing interests: $\mathrm{SB}, \mathrm{AS}$ and $\mathrm{AB}$ : received investigator fee for conduct of the study; RJ, KD and $\mathrm{VK}$ are paid employees of Wockhardt Ltd.

\section{REFERENCES}

1. Mao JS, Dong DX, Zhang HY, et al. Primary study of attenuated live hepatitis A vaccine (H2 strain) in humans. J Infect Dis. 1989;159:621-24.

2. Zhuang FC, Qian W, et al. Persistent efficacy of live attenuated hepatitis A vaccine (H2-strain) after a mass vaccination program. Chin Med J (Engl). 2005;118:1851-856.

3. Zhuang FC, Mao ZA, Jiang LM, et al. Long term immunogenicity and effectiveness of live attenuated hepatitis A vaccine (H2-strain) - A study on the result of 15 years' follow up. Zhonghua Liu Xing Bing Xue Za Zhi. 2010;31:1332-335.

4. Shah N, Faridi MMA, Mitra M, et al. Review of long term immunogenicity and tolerability of live hepatitis A vaccine. Hum Vaccin Immunother. 2020 Apr 3;1-6

5. WHO position paper on hepatitis A vaccines - June, 2012. Weekly Epidemiological Record. 2012;87:261-76.

6. Pemde H. Hepatitis A vaccines. In: Advisory Committee on Vaccines and Immunization Practices, Indian Academy of Pediatrics. IAP Guidebook on Immunization 20182019. 3rd edition. Jaypee Brothers Medical Publishers; 2020. p. 265-78.

7. Bhave S, Bavdekar A, Madan Z, et al. Evaluation of 
immunogenicity and tolerability of a live attenuated hepatitis A vaccine in Indian children. Indian Pediatr. 2006;43:983-7

8. Bhave S, Sapru A, Bavdekar A, Bawangade S, Pandit A. Immunogenicity of single dose live attenuated hepatitis A vaccine. Indian Pediatr. 2011;48:135-37.

9. Bhave S, Sapru A, Bavdekar A, Kapatkar V, Mane A. Long-term immunogenicity of single dose of live attenuated hepatitis A vaccine in Indian children. Indian Pediatr. 2015; 52:687-90.

10. Faridi MMA, Shah N, Ghosh TK, et al. Immunogenicity and safety of live attenuated hepatitis A vaccine: A multicentric study. Indian Pediatr. 2009;46:29-34.

11. Mitra M, Shah N, Faridi MMA, et al. Long term follow-up study to evaluate immunogenicity and safety of a single dose of live attenuated hepatitis A vaccine in children. Hum
Vaccin Immunother. 2015;11:1147-152.

12. Wang $\mathrm{XY}, \mathrm{Xu} \mathrm{ZY}$, Ma JC, et al. Long term immunogenicity after single and booster dose of a live attenuated hepatitis A vaccine: Results from 8-year followup. Vaccine. 2007; 25:446-49.

13. Zhang QY, Chen H, Lin Z, Lin JM. Comparison of chemiluminescence enzyme immunoassay based on magnetic microparticles with traditional colorimetric ELISA for the detection of serum $\alpha$-fetoprotein. J Pharm Analysis. 2012;2:130-35.

14. Arankalle V, Mitra M, Bhave S, et al. Changing epidemiology of hepatitis A virus in Indian children. Vaccine: Development and Therapy. 2014;4:7-13.

15. Chen Y, Zhou C-L, Zhang X-J, et al. Immune memory at 17-years of follow up of a single dose of live attenuated hepatitis A vaccine. Vaccine. 2018;36:114-21. 